\title{
Silicone Granulomas in the Setting of Acupuncture with Silicone-Coated Needles
}

\section{Introduction}

Silicone is widely used in various capacities in medicine including aesthetic and prosthetic joint implants as well as in the coating of vials, catheters, drains, shunts, and needles [1]. Silicone-coated needles have become increasingly popular in alternative medicine techniques such as acupuncture to ease the insertion of needles. It has been well established that silicone can lead to a foreign body granulomatous reaction in the skin with the first histopathologic cases reported in 1964 [2]. Herein, we present what is believed to be the third reported case of silicone granulomas secondary to silicone-coated acupuncture needles $[3,4]$.

\section{Report of a Case}

A 25-year-old Caucasian female presented with a 6-month history of a mildly pruritic rash of the radial wrists, left medial knee and dorsal feet. Individual lesions were stable in size and location for months and treatment with $1 \%$ hydrocortisone cream was unsuccessful. Physical exam revealed grouped, 1-2 mm, shiny, erythematous to violaceous papules on the radial wrists (Figure 1), left medial knee and the medial dorsal feet.

Punch biopsy was performed revealing focal attenuation of the epidermis with underlying epidermal epithelial granulomas with a prominent cuff of lymphocytes (Figure 2). No polarizable foreign material was identified. Acid-fast bacilli, Grocott's methenamine silver and Warthin Starry stains were all negative. The extent of lymphocytic cuffing was more extensive than typically observed in sarcoidosis, but pathology alone could not rule out sarcoidosis or an infectious etiology.

On follow up, a pulmonology evaluation to rule out systemic sarcoidosis was discussed. However, during this discussion the patient

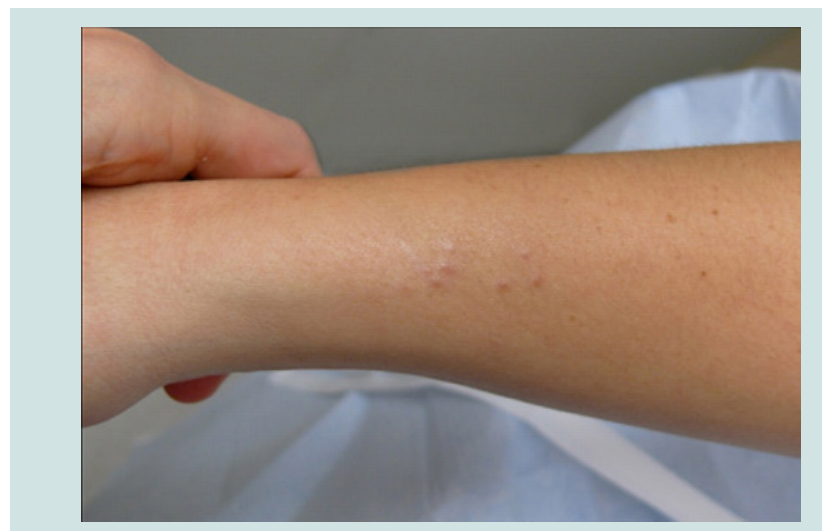

Figure 1: Grouped, 1-2 mm, shiny, erythematous to violaceous papules on the right radial wrist.

\section{Clinical \& Investigative Dermatology}

\author{
Amanda J. Tschetter ${ }^{1 *}$, Phillip C. Hochwalt ${ }^{1}$, Erica \\ Colleran $^{3}$ and Mary S. Stone ${ }^{1,2}$ \\ ${ }^{1}$ University of Iowa, Department of Dermatology, Iowa City, IA, USA \\ ${ }^{2}$ University of Iowa, Department of Pathology, Iowa City, IA, USA \\ ${ }^{3}$ Iowa City Dermatology, Iowa City, IA, USA
}

\section{*Address for Correspondence}

Amanda J. Tschetter, 200 Hawkins Drive, 40024 PFP, Iowa City, IA 52242, USA, Email: Amanda-Tschetter@uiowa.edu

\section{Submission: 15 November 2013}

Accepted: 15 January 2014

Published: 17 January 2014

Reviewed \& Approved by: Dr. Nihal Ahmad

Professor, Department of Dermatology

University of Wisconsin, USA

\section{A. Original magnification $\times 10$}

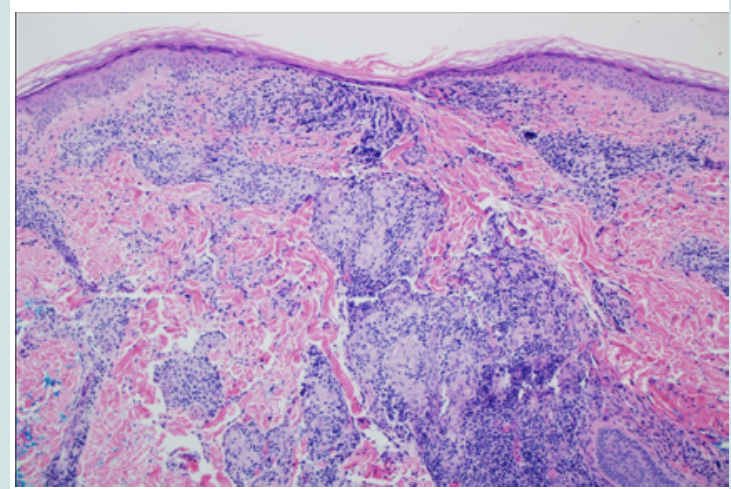

B. Original magnification $\times 40$

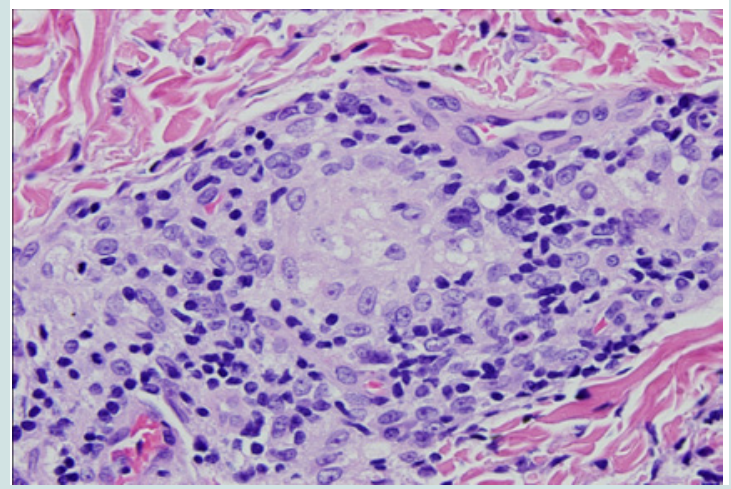

Figure 2: Histopathologic specimens under hematoxylin-eosin stain show focal attenuation of the epidermis with underlying epidermal epithelial granulomas with a prominent cuff of lymphocytes and macrophage vacuolization.

admitted to undergoing acupuncture 1 month prior to the onset of the eruption which broadened the differential to include non-sarcoid foreign body granulomas. The acupuncturist was contacted and the use of silicone-coated needles was confirmed. Given the lack of systemic symptoms along with the fact that the clinical appearance, timing of the eruption, and pathologic appearance were all consistent with 
Citation: Tschetter AJ, Hochwalt PC, Colleran E, Stone MS. Silicone Granulomas in the Setting of Acupuncture with Silicone-Coated Needles. J Clin Investigat Dermatol. 2014;2(1): 2.

silicone granulomas, a pulmonology evaluation was deferred. As the eruption was essentially asymptomatic and the patient unbothered by the appearance, treatments were also deferred. The patient returned to clinic approximately 3 months later for a different problem and it was noted that the granulomatous skin lesions remained unchanged at that time.

\section{Discussion}

To our knowledge, this is the third reported case of silicone granulomas secondary to acupuncture with silicone-coated needles. The first reported case was that of a 55-year-old Japanese female who developed silicone granulomas at entry points of acupuncture, venipuncture and skin biopsy sites. Silicone was confirmed to be the causative agent by analytical electron microscopy with an energydispersive X-ray detector [3]. In 2001 a second case was reported in a 59 -year-old Caucasian female. The diagnosis was based on clinicopathologic correlation [4].

While granulomatous reactions to foreign material in the skin most commonly occur as an isolated finding, it is important to note that the histopathologic presence of this finding does not exclude a diagnosis of systemic sarcoidosis [5]. Specific and nonspecific cutaneous manifestations of sarcoidosis, defined by the presence or absence of noncaseating granulomas, respectively, are present in 20 $35 \%$ cases of sarcoidosis. The wide range of cutaneous morphologies may precede, coincide with, or develop after a diagnosis of systemic sarcoidosis. Unfortunately, the corresponding frequencies are unknown [6,7]. It is well established that sarcoidosis frequently localizes to sites of trauma including tattoo, surgery, venipuncture, vaccination and purified protein derivative testing sites [8]. A series by Marcoval et al. observed sarcoid-specific skin lesions in 15\% of sarcoidosis cases. Of those, $22 \%$ demonstrated foreign body material within the cutaneous granulomas, most of which were located in trauma-prone areas such as the extensor extremities [9]

Given this information, it cannot be stated with certainty that the patient described herein will not develop systemic sarcoidosis. In fact, many postulate that the inflammatory cascade leading to sarcoidal granulomas is set off by exposure to certain extrinsic antigens in genetically susceptible individuals [6]. While several potential antigens exist, occupational/environmental exposure to foreign materials such as silicone may be implicated in rare cases [10]. Additionally, a lack of systemic symptoms at the time of presentation does not preclude underlying sarcoidosis. Various series have estimated that $30-50 \%$ of sarcoidosis patients are asymptomatic at the time of diagnosis [11]. While we feel that the risk of sarcoidosis in our patient is quite low, monitoring for development of additional symptoms is warranted.

The primary aim of this paper is to alert physicians to a potential complication of acupuncture, an alternative medicine practice that continues to gain popularity in the United States. Acupuncture is performed by varying providers including acupuncturists, dentists, and physicians for a vast array of ailments. The National Health Interview Survey reports that the use of acupuncture in the U.S. increased by approximately one million people from 2002 to 2007 [12]. $6.5 \%$ of those surveyed had undergone at least one acupuncture session [13]. In addition to acupuncture needles, most hypodermic needles and syringes are coated with silicone as injection with such needles is noted to be less painful [1]. It is unclear why some people are more susceptible to forming silicone granulomas and whether the risk is increased with a longer single exposure time or repeated exposures in the same location. Clinicians and pathologists alike should be aware of this potential side effect of acupuncture as there will likely be an increased number of silicone granuloma cases as acupuncture utilization in the U.S. continues to grow.

\section{References}

1. Ratner BD (2004) Biomaterials science: an introduction to materials in medicine. 2nd Edition, Elsevier Academic Press.

2. Winer LH, Sternberg TH, Lehman R, Ashley FL (1964) Tissue Reactions to Injected Silicone Liquids. A Report of Three Cases. Arch Dermatol 90: 588593.

3. Yanagihara M, Fujii T, Wakamatu N, Ishizaki H, Takehara T, et al. (2000) Silicone granuloma on the entry points of acupuncture, venepuncture and surgical needles. J Cutan Pathol 27: 301-305.

4. Alani RM, Busam K (2001) Acupuncture granulomas. J Am Acad Dermatol 45: S225-226.

5. Walsh NM, Hanly JG, Tremaine R, Murray S (1993) Cutaneous sarcoidosis and foreign bodies. Am J Dermatopathol 15: 203-207.

6. Haimovic A, Sanchez M, Judson MA, Prystowsky S (2012) Sarcoidosis: a comprehensive review and update for the dermatologist: part I. Cutaneous disease. J Am Acad Dermatol 66: 699.e1-18.

7. Ghosh UC, Ghosh SK, Hazra K, Mondal A (2013) Ichthyosiform sarcoidosis revisited. Indian J Dermatol Venereol Leprol 79: 795-798.

8. Val-Bernal JF, Sanchez-Quevedo MC, Corral J, Campos A (1995) Cutaneous sarcoidosis and foreign bodies. An electron probe roentgenographic microanalytic study. Arch Pathol Lab Med 119: 471-474.

9. Marcoval J, Mana J, Moreno A, Gallego I, Fortuno Y, et al. (2001) Foreign bodies in granulomatous cutaneous lesions of patients with systemic sarcoidosis. Arch Dermatol 137: 427-430.

10. Newman KL, Newman LS (2012) Occupational causes of sarcoidosis. Curr Opin Allergy Clin Immunol 12: 145-150.

11. Costabel U, Guzman J, Baughman RP (2007) Systemic evaluation of a potential cutaneous sarcoidosis patient. Clin Dermatol 25: 303-311.

12. Acupuncture: An Introduction. 2013 National Center for Complementary and Alternative Medicine 2011; National Institute of Health.

13. National Health Interview Survey. Adult Alternative Medicine 2007; Center for Disease Control:Center for Disease Control.

\section{Copyright}

(c) 2014 Tschetter AJ, et al. This is an open access article distributed under the Creative Commons Attribution License, which permits unrestricted use, distribution, and reproduction in any medium, provided the original work is properly cited. 